\title{
Liverpool Telescope 2: beginning the design phase
}

\author{
Christopher M. Copperwheat ${ }^{\mathrm{a}}$, Iain A. Steele ${ }^{\mathrm{a}}$, Robert M. Barnsley ${ }^{\mathrm{a}, \mathrm{b}}$, Stuart D. Bates ${ }^{\mathrm{a}}$, Mike \\ F. Bode ${ }^{\mathrm{a}}$, Neil R. Clay ${ }^{\mathrm{a}}$, Chris A. Collins ${ }^{\mathrm{a}}$, Helen E. Jermak ${ }^{\mathrm{a}}$, Johan H. Knapen ${ }^{\mathrm{c}, \mathrm{d}}$, Jon M. \\ Marchant $^{\mathrm{a}}$, Chris J. Mottram ${ }^{\mathrm{a}}$, Andrzej S. Piascik ${ }^{\mathrm{a}}$, and Robert J. Smith ${ }^{\mathrm{a}}$ \\ ${ }^{a}$ Astrophysics Research Institute, Liverpool John Moores University, Liverpool Science Park, \\ 146 Brownlow Hill, Liverpool, L3 5RF, UK \\ ${ }^{b}$ Department of Physics, University of Oxford, Denys Wilkinson Building, Keble Road, Oxford \\ OX1 3RH, UK \\ 'Instituto de Astrofísica de Canarias, E-38200 La Laguna, Tenerife, Spain \\ ${ }^{\mathrm{d}}$ Departamento de Astrofísica, Universidad de La Laguna, E-38205 La Laguna, Tenerife, Spain
}

\begin{abstract}
The Liverpool Telescope is a fully robotic 2-metre telescope located at the Observatorio del Roque de los Muchachos on the Canary Island of La Palma. The telescope began routine science operations in 2004, and currently seven simultaneously mounted instruments support a broad science programme, with a focus on transient followup and other time domain topics well suited to the characteristics of robotic observing. Work has begun on a successor facility with the working title 'Liverpool Telescope 2'. We are entering a new era of time domain astronomy with new discovery facilities across the electromagnetic spectrum, and the next generation of optical survey facilities such as LSST are set to revolutionise the field of transient science in particular. The fully robotic Liverpool Telescope 2 will have a 4-metre aperture and an improved response time, and will be designed to meet the challenges of this new era. Following a conceptual design phase, we are about to begin the detailed design which will lead towards the start of construction in 2018, for first light $\sim 2022$. In this paper we provide an overview of the facility and an update on progress.
\end{abstract}

Keywords: Liverpool Telescope, Liverpool Telescope 2, Time domain astronomy, Robotic telescopes, Optical/infrared spectroscopy, Supernovae, Gamma-ray bursts, Gravitational waves

\section{INTRODUCTION}

The Liverpool Telescope ${ }^{1}$ (LT) is a fully robotic optical/near-infrared telescope with a Ritchey-Chrétien Cassegrain design and a 2-metre clear aperture. The telescope is located at the Observatorio del Roque de los Muchachos on the Canary Island of La Palma, and is owned and operated by the Astrophysics Research Institute of Liverpool John Moores University (LJMU). It was designed and constructed on Merseyside in the UK by Telescope Technologies Limited, a spin-off company of the university. First light for the telescope was in July 2003, with routine robotic science operations beginning in 2004. Robotic telescopes are well suited to the study of the time variable sky, since they are capable of rapid reaction to unpredictable targets-of-opportunity, and well as flexible scheduling of observations for the monitoring of variable objects on a wide range of timescales. The operational life of the LT has coincided with the beginnings of the era of wide field synoptic surveys such as the Palomar Transient Factory ${ }^{2}$ (PTF), as well as the Swift ${ }^{3}$ mission, which has opened up the field of gamma-ray burst science. The rapid and flexible response of the LT has made it an ideal tool for the exploitation of new transients discovered by these and other facilities. ${ }^{4-7}$

Development began in 2012 on an LT successor facility, with the working title 'Liverpool Telescope 2' (LT2). It was recognised that the next generation of optical synoptic survey facilities such as LSST ${ }^{8}$ will bring about a new era in transient science, which drives the need for new follow-up telescopes for exploitation. LSST will not only report transients at much fainter magnitudes, but also observe with a cadence such that much of the long

Further author information:

C.M.C.: E-mail: c.m.copperwheat@ljmu.ac.uk, Telephone: +44 1512312914

Ground-based and Airborne Telescopes VI, edited by Helen J. Hall, Roberto Gilmozzi, Heather K. Marshall, Proc. of SPIE Vol. 9906, 99063A · C 2016 SPIE · CCC code: 0277-786X/16/\$18 · doi: 10.1117/12.2231755 
term photometric monitoring currently provided by follow-up telescopes like the LT will be provided 'for free' by the survey telescope itself. The field of gamma-ray burst science will continue with facilities such as SVOM ${ }^{9}$ succeeding Swift. For follow-up, potentially the biggest science gains will be realised by more rapid reaction to triggers from discovery facilities. However perhaps the most exciting development in time domain science in recent years is the detection of gravitational waves,${ }^{10}$ opening an entirely new window onto the universe. The next milestone will be the discovery of the electromagnetic counterpart to such an event, and there is great potential for a new breed of robotic telescope to contribute to this developing field.

In 2016 the LT2 project is moving into its second phase, during which we will produce a detailed design for the telescope and complete the funding consortium. The aim is to begin construction in 2018 with full science operations following in $\sim 2022$. In this paper we provide an overview of the goals and current status of the project.

\section{KEY SCIENCE DRIVERS}

The LT is a versatile facility. One of its core strengths is its diverse instrument payload, which enables a wide variety of science topics to be pursued. Historically, new instrument concepts have also been brought to the telescope on short timescales, which has helped maintain the LT's status as a world-leading time domain telescope. We aim to maintain this flexibility with the new telescope, and in the LT2 science white paper ${ }^{11}$ we list a wide selection of time domain topics in the next decade to which it may make a contribution. However, we identify three core priorities, which we briefly discuss here.

\subsection{Exploitation of the next generation of ground-based synoptic surveys}

The study of explosive transients such as supernovae has been revolutionised by the current generation of widefield, ground-based, so-called 'synoptic' surveys, such as iPTF' ${ }^{2}$ and Pan-STARRS. ${ }^{12}$ These surveys have been important for the discovery of new subclasses such as the superluminous supernovae, but also for providing more statistically complete samples of known classes such as supernovae Type Ia. A current issue is the lack of capacity for follow-up of the wide field surveys: a small fraction of newly discovered transients receive a spectroscopic classification or any detailed exploitation. The $\mathrm{PESSTO}^{13}$ survey has demonstrated that dedicating large amounts of telescope time to spectroscopic follow-up can be extremely productive, and the flexible scheduling capability of robotic telescopes such as the LT makes it straightforward to obtain well-sampled observations of large numbers of targets, and a rapid reaction capability can be very important for SNe discovered very soon after the explosion. The picture will change somewhat in the LSST era however: the monitoring cadences of the next generation of surveys means that much of the photometric monitoring will be provided by the survey itself. However, the spectroscopic follow-up problem will worsen, since the rate of transient alerts provided to the community will increase by orders of magnitude. LSST will also probe the transient sky to unprecedented depths, meaning a 4-metre aperture is likely the minimum for effective follow-up.

\subsection{Gamma-ray bursts: beyond the Swift era}

Gamma-ray bursts (GRBs) are the most energetic explosions to be detected in the universe. They are characterised by a short and intense burst of prompt gamma-ray emission which is commonly followed by a rapidly fading (minutes to hours) afterglow detectable at X-ray and optical wavelengths. The success of this science area in the past decade has been driven by rapid response: the Swift Gamma-Ray Burst Mission ${ }^{3}$ and the Fermi Gamma Ray Space Telescope ${ }^{14}$ provide triggers to ground-based follow-up facilities with extremely short latencies, and those follow-up telescopes typically immediately override their active observation in response to the trigger. The LT is an ideal tool for this role due to its rapid reaction capability and diverse instrument suite enabling a flexible response. ${ }^{15}$ Both Swift (launched 2004) and Fermi (launched 2008) are in the extended phases of their missions, and so we look now to the post-Swift era. The leading successor mission is is the joint French-Chinese observatory SVOM. ${ }^{9}$ This facility will combine a space-based gamma-ray and X-ray detector with a ground based network of small telescopes for afterglow localisation and redshift determination. There is still a role for larger aperture facilities for detailed follow-up, but building on previous successes is not merely a case of increasing aperture: the rapid decay of the afterglow means that a telescope with a greater slew speed can collect more photons than one with a significantly larger aperture. 


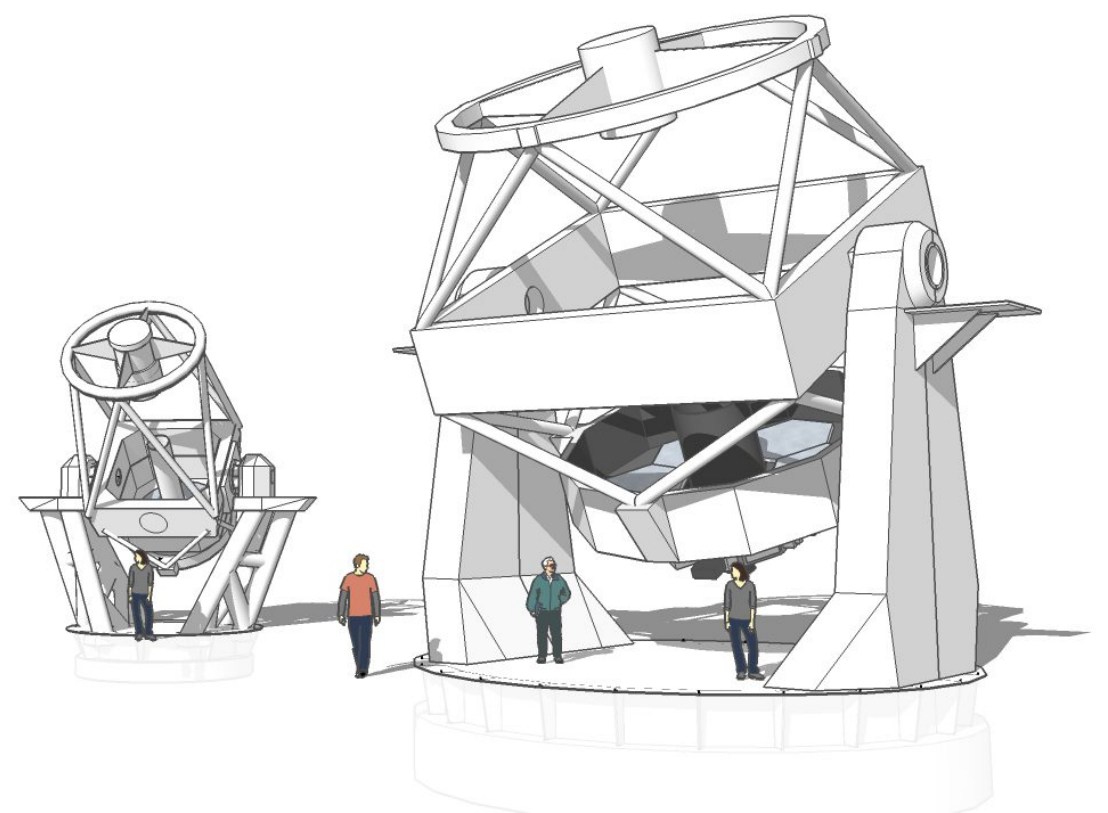

Figure 1. Design concept for Liverpool Telescope 2, alongside the existing Liverpool Telescope

\subsection{Counterparts of gravitational wave sources}

The detection of gravitational waves during the first Advanced LIGO (aLIGO) science run ${ }^{10}$ is one of the most exciting moments in physics that we will see in our lifetimes. The origin of the event was the merger of two massive black holes, but as detector sensitivity increases and a larger volume of space is explored, the majority of detections are expected to be neutron star merger events. The potential of this new era of gravitational wave science is immense: a new window has been opened on the universe. A neutron star event should produce an associated electromagnetic counterpart, and the detection of this counterpart is the next milestone. The LT is an active participant in the observing campaigns running alongside the aLIGO runs with the aim of making this detection. ${ }^{16}$ A significant challenge is the large positional uncertainty of any GW detection, which is of the order of hundred of square degrees now and will be at best a few square degrees when the detectors reach full sensitivity in the next decade. Survey telescopes with wide fields-of-view are required for the transient search, but the real problem is that a sky area of this size will contain many unrelated variable candidates alongside the true counterpart. This drives similar requirements to the optical surveys follow-up case (Section 2.1): systematic classification and follow-up is required to distinguish the true counterpart from the more mundane transient sources, and then a range of facilities will be employed for the detailed exploitation.

\section{BEGINNING THE DESIGN PHASE}

\subsection{Conceptual design}

The conceptual design phase resulted in a baseline list of technical requirements. ${ }^{11,17}$ Based on an assumed brightness distribution of our expected targets and an assessment of our likely instrument payload, we specify a clear aperture of at least 4 metres for LT2. Our second core requirement is the capability for a world leading response to an electronically transmitted alert from another facility. Rapid reaction is the core strength of robotic observing, and so it makes sense to emphasise this attribute when designing a next generation robotic facility. This is potentially not just important for fast fading transients (such as GRB afterglows), for exploring the faint/fast region of the transient phase space which will be opened up by LSST, but also for reducing observational overheads and maintaining efficiency during large scale programmes of systematic classification and exploitation. 
This rapid reaction requirement has a fundamental impact on the overall telescope design. We commissioned a number of preliminary optical design studies which advocated a Ritchey-Chrétien design with a fast primary mirror: $f / 1$ or $f / 1.5$. There are a range of options for the final focal ratio at this stage, with $f / 8$ somewhere in the middle of that range. The result is a telescope with a 4-metre aperture and a primary-secondary separation which is comparable to the 2-metre LT. The resulting low moment of inertia facilitates fast slewing. The studies also proposed a segmented primary mirror, which significantly reduces the mirror mass. We have considered a number of different configurations for a segmented mirror, but a 6 -segment mirror is potentially the best option. Earlier in development an 18-segment configuration was preferred, ${ }^{17}$ but 6 offers a number of advantages: it means all the segments are identical, which simplifies production; the segments are larger, which means we do not need to phase the mirror in order to be seeing-limited at infrared wavelengths; and the segments would be the same size as those used on the GTC telescope, so an appropriate realuminising facility already exists at the observatory.

As well as segmenting the mirror, it is expected that the majority of the instruments will be mounted on Nasmyth platforms rather than Cassegrain, again to reduce the load on the altitude axis. We envisage also a modern, lightweight structure employing modern materials to reduce overall mass as much as possible while maintaining stiffness. The enclosure will likely be of a similar clamshell design to the successful LT enclosure, which removes the problem of dome seeing while providing access to the entire sky, facilitating rapid reaction.

\subsection{Site, and a new role for the LT}

A number of sites were considered, but it has been decided that LT2 will be co-located with LT at the Observatorio del Roque de los Muchachos on the Canary island of La Palma. This is a world-class northern site at a latitude of $28^{\circ}$, providing good access to the southern sky. Specifically, the former site of the Automatic Transit Circle has become available, and has been offered by the Instituto de Astrofísica de Canarias (IAC) as part of their contribution to the project. Having both telescopes on the same site offers interesting scientific possibilities. With LT2 expected to focus on spectroscopic follow-up, we intend to replace the instrument suite on the LT with a single prime-focus imager. This camera would be equipped with a filter wheel and would provide a total on-sky area of $\sim 2.8 \mathrm{deg}^{2}$. This instrument would be used for surveys, for the localisation of transients with large positional uncertainties (such as gravitational wave counterparts) and for educational work via the National Schools Observatory.

\subsection{Next steps}

2016 will see the appointment at LJMU of the engineering and science staff which will form the core of the project office for the next phase. The project office will develop the current outline specification and concept into a fully realised design. The aim is that this detailed design, along with the associated external reviews, will be completed by 2018 , with the project funding consortium nearing completion on a similar timeline. Construction will then begin for an anticipated commencement of science operations in 2022. This timeline is matched to the beginning of operations for key facilities such as LSST and the completed gravitational wave detector network: facilities which we anticipate will be a major source of targets for LT2. The IAC will also be appointing dedicated engineering staff in 2016 to work on the project, and one of the major initial tasks for the project office will be the outlining of work packages for the project partners, although the degree to which design work will be centralised is currently uncertain and will depend to some degree on the final make-up of the consortium.

\section{INSTRUMENTATION}

One of the main strengths of the LT is its diverse instrument suite. The instrumentation is all mounted on the Cassegrain station, with a deployable fold mirror allowing for rapid and automated instrument changes. A large selection of instrumentation not only provides a flexible and diverse scientific capability, but also helps maintain a low overall technical downtime statistic, since modern astronomical instrumentation is necessarily complicated and often amongst the most unreliable subsystems. For these reasons, we anticipate that LT2 will have at least five focal stations for the simultaneous mounting of a diverse array of instrumentation, although as noted in Section 3.1 our initial design studies suggest that for mass-balance reasons we may choose to mount the majority of instruments on Nasmyth platforms, with at most one instrument on the Cassegrain station. 
At the centre of the LT2 science case is the need for spectroscopic follow-up of transient objects, therefore it is anticipated the primary instrument will be a spectrograph. The ESO X-shooter instrument ${ }^{18}$ is a demonstration of the power of a wide wavelength range, and this is particularly important for transient follow-up in which there may be a very short temporal window in order to obtain the required observations. The wavelength range will extend into the near-infrared, although the site is not well suited to observations beyond the $H$-band. The design of the instrument is undecided at this stage. The main spectrograph on the LT is bench mounted and fibre-fed, however the more recent SPRAT spectrograph, ${ }^{19}$ designed specifically for transient classification, is of a long-slit design, and achieves a much higher throughput. It was thought that robotic acquisition of the target onto a narrow slit would be a technical issue, but SPRAT has demonstrated that reliable and consistent target acquisition can be achieved via an iterative WCS fit. On the other hand, the use of an IFU potentially leads to a more rapid acquisition, which feeds into the LT2 rapid reaction paradigm. On the question of resolution, the LT2 science case suggests an intermediate resolving power of the order of a few thousand is sufficient for most of the science we wish to pursue. However, SPRAT and similar instrument have demonstrated the power and popularity of a low resolution, $R \sim 300$ spectrograph for transient classification, and this will continue to be the case in the LSST era. Ideally the LT2 spectrograph would offer both modes.

High-cadence photometry; for exoplanet transits, eclipsing binaries and GRB afterglow light curves; is an important part of the LT instrument complement, and this is likely to also be true for LT2. While LSST will provide lightcurves with cadences of the order of days, the study of rapid variability on timescales down to fractions of a second will still require follow-up telescopes such as LT2. For GRB follow-up in particular perhaps the exemplar instrument in the current era is the GROND camera ${ }^{20}$ mounted on the MPI/ESO $2.2 \mathrm{~m}$ at the La Silla observatory in Chile. The argument for simultaneous multiband coverage is the same as the argument for a wide wavelength range for the spectrograph: when observing a fast-fading or evolving transient, there may be no time for filter changes. The potential of high cadence photometry has been enhanced by a number of technical advances in recent years, in particular the advent of Scientific-CMOS (sCMOS) cameras, which can combine low noise with high readout speeds, and do not suffer the charge multiplication noise that effectively reduces the quantum efficiency of the electron multiplying CCDs used in a number of current high cadence instruments. We are currently evaluating the use of these detectors for LT and LT2 instrumentation, and in 2016 a PhD student will begin development work on a prototype instrument (Steele et al., these proceedings). These detectors also have much potential for polarimetric applications, and polarimetry has been a particular strength of the LT instrument complement, producing a number of unique results. ${ }^{5-7} \mathrm{~A}$ design for a next generation polarimeter has been developed, for potential deployment on LT or LT2 (Jermak et al., these proceedings).

Looking further ahead, a technology that is particularly interesting for our science goals are Kinetic Inductance Detectors (KIDs). These detectors can measure both the energy and arrival time of individual photons, which makes them a powerful tool for time domain science, and in particular the follow-up of fast evolving transients in which the ideal monitoring cadences may not be known prior to detection. Large arrays of such detectors are now possible, and concepts exist for highly efficient, medium spectral resolution wide band-pass astronomical spectrographs employing such arrays. ${ }^{21}$

\section{SUMMARY}

The Liverpool Telescope is a mature facility with over a decade of successful science operations, covering a diverse programme with a focus on time domain science. We are in the development stages of a new telescope, codenamed 'Liverpool Telescope 2' which will build on this success with a modern robotic facility designed to meet the needs of the new era of time domain astronomy which will be spearheaded by LSST. LT2 will be a 4-metre, fully robotic telescope with a diverse instrument suite but a focus on rapid spectroscopic classification and exploitation of new transient sources. In 2016 we begin the detailed design phase, which will build towards the star of construction in 2018, with a view to beginning full science operations in 2022.

\section{ACKNOWLEDGMENTS}

The Liverpool Telescope is operated on the island of La Palma by Liverpool John Moores University in the Spanish Observatorio del Roque de los Muchachos of the Instituto de Astrofísica de Canarias with financial support 
from the UK Science and Technology Facilities Council. Liverpool Telescope 2 is currently being developed in partnership between the Astrophysics Research Institute of Liverpool John Moores University and the Instituto de Astrofísica de Canarias. JHK acknowledges financial support from the Spanish Ministry of Economy and Competitiveness (MINECO) under grant number AYA2013-41243-P, and thanks the Astrophysics Research Institute of Liverpool John Moores University for their hospitality, and the Spanish Ministry of Education, Culture and Sports for financial support of his visit there, through grant number PR2015-00512.

\section{REFERENCES}

[1] Steele, I. A., Smith, R. J., Rees, P. C., Baker, I. P., Bates, S. D., Bode, M. F., Bowman, M. K., Carter, D., Etherton, J., Ford, M. J., Fraser, S. N., Gomboc, A., Lett, R. D. J., Mansfield, A. G., Marchant, J. M., Medrano-Cerda, G. A., Mottram, C. J., Raback, D., Scott, A. B., Tomlinson, M. D., and Zamanov, R., "The Liverpool Telescope: performance and first results," in [Ground-based Telescopes], Oschmann, Jr., J. M., ed., Society of Photo-Optical Instrumentation Engineers (SPIE) Conference Series 5489, 679-692 (Oct. 2004).

[2] Rau, A., Kulkarni, S. R., Law, N. M., Bloom, J. S., Ciardi, D., Djorgovski, G. S., Fox, D. B., Gal-Yam, A., Grillmair, C. C., Kasliwal, M. M., Nugent, P. E., Ofek, E. O., Quimby, R. M., Reach, W. T., Shara, M., Bildsten, L., Cenko, S. B., Drake, A. J., Filippenko, A. V., Helfand, D. J., Helou, G., Howell, D. A., Poznanski, D., and Sullivan, M., "Exploring the Optical Transient Sky with the Palomar Transient Factory," PASP 121, 1334-1351 (Dec. 2009).

[3] Gehrels, N., Chincarini, G., Giommi, P., Mason, K. O., Nousek, J. A., Wells, A. A., White, N. E., Barthelmy, S. D., Burrows, D. N., Cominsky, L. R., Hurley, K. C., Marshall, F. E., Mészáros, P., Roming, P. W. A., Angelini, L., Barbier, L. M., Belloni, T., Campana, S., Caraveo, P. A., Chester, M. M., Citterio, O., Cline, T. L., Cropper, M. S., Cummings, J. R., Dean, A. J., Feigelson, E. D., Fenimore, E. E., Frail, D. A., Fruchter, A. S., Garmire, G. P., Gendreau, K., Ghisellini, G., Greiner, J., Hill, J. E., Hunsberger, S. D., Krimm, H. A., Kulkarni, S. R., Kumar, P., Lebrun, F., Lloyd-Ronning, N. M., Markwardt, C. B., Mattson, B. J., Mushotzky, R. F., Norris, J. P., Osborne, J., Paczynski, B., Palmer, D. M., Park, H.-S., Parsons, A. M., Paul, J., Rees, M. J., Reynolds, C. S., Rhoads, J. E., Sasseen, T. P., Schaefer, B. E., Short, A. T., Smale, A. P., Smith, I. A., Stella, L., Tagliaferri, G., Takahashi, T., Tashiro, M., Townsley, L. K., Tueller, J., Turner, M. J. L., Vietri, M., Voges, W., Ward, M. J., Willingale, R., Zerbi, F. M., and Zhang, W. W., "The Swift Gamma-Ray Burst Mission," ApJ 611, 1005-1020 (Aug. 2004).

[4] Nugent, P. E., Sullivan, M., Cenko, S. B., Thomas, R. C., Kasen, D., Howell, D. A., Bersier, D., Bloom, J. S., Kulkarni, S. R., Kandrashoff, M. T., Filippenko, A. V., Silverman, J. M., Marcy, G. W., Howard, A. W., Isaacson, H. T., Maguire, K., Suzuki, N., Tarlton, J. E., Pan, Y.-C., Bildsten, L., Fulton, B. J., Parrent, J. T., Sand, D., Podsiadlowski, P., Bianco, F. B., Dilday, B., Graham, M. L., Lyman, J., James, P., Kasliwal, M. M., Law, N. M., Quimby, R. M., Hook, I. M., Walker, E. S., Mazzali, P., Pian, E., Ofek, E. O., Gal-Yam, A., and Poznanski, D., "Supernova SN 2011fe from an exploding carbon-oxygen white dwarf star," Nature 480, 344-347 (Dec. 2011).

[5] Mundell, C. G., Steele, I. A., Smith, R. J., Kobayashi, S., Melandri, A., Guidorzi, C., Gomboc, A., Mottram, C. J., Clarke, D., Monfardini, A., Carter, D., and Bersier, D., "Early Optical Polarization of a Gamma-Ray Burst Afterglow," Science 315, 1822 (Mar. 2007).

[6] Steele, I. A., Mundell, C. G., Smith, R. J., Kobayashi, S., and Guidorzi, C., "Ten per cent polarized optical emission from GRB090102," Nature 462, 767-769 (Dec. 2009).

[7] Mundell, C. G., Kopač, D., Arnold, D. M., Steele, I. A., Gomboc, A., Kobayashi, S., Harrison, R. M., Smith, R. J., Guidorzi, C., Virgili, F. J., Melandri, A., and Japelj, J., "Highly polarized light from stable ordered magnetic fields in GRB 120308A," Nature 504, 119-121 (Dec. 2013).

[8] Ivezic, Z., Tyson, J. A., Abel, B., and Acosta, E., "LSST: from Science Drivers to Reference Design and Anticipated Data Products," ArXiv e-prints (May 2008).

[9] Paul, J., Wei, J., Basa, S., and Zhang, S.-N., "The Chinese-French SVOM mission for gamma-ray burst studies," Comptes Rendus Physique 12, 298-308 (Apr. 2011).

[10] Abbott, B. P., Abbott, R., Abbott, T. D., Abernathy, M. R., Acernese, F., Ackley, K., Adams, C., Adams, T., Addesso, P., Adhikari, R. X., and et al., "Observation of Gravitational Waves from a Binary Black Hole Merger," Physical Review Letters 116, 061102 (Feb. 2016). 
[11] Copperwheat, C. M., Steele, I. A., Barnsley, R. M., Bates, S. D., Bersier, D., Bode, M. F., Carter, D., Clay, N. R., Collins, C. A., Darnley, M. J., Davis, C. J., Gutierrez, C. M., Harman, D. J., James, P. A., Knapen, J. H., Kobayashi, S., Marchant, J. M., Mazzali, P. A., Mottram, C. J., Mundell, C. G., Newsam, A., Oscoz, A., Palle, E., Piascik, A., Rebolo, R., and Smith, R. J., "Liverpool telescope 2: a new robotic facility for rapid transient follow-up," Experimental Astronomy 39, 119-165 (Mar. 2015).

[12] Kaiser, N., Burgett, W., Chambers, K., Denneau, L., Heasley, J., Jedicke, R., Magnier, E., Morgan, J., Onaka, P., and Tonry, J., "The Pan-STARRS wide-field optical/NIR imaging survey," in [Ground-based and Airborne Telescopes III], SPIE 7733, 77330E (July 2010).

[13] Smartt, S. J., Valenti, S., Fraser, M., Inserra, C., Young, D. R., Sullivan, M., Pastorello, A., Benetti, S., Gal-Yam, A., Knapic, C., Molinaro, M., Smareglia, R., Smith, K. W., Taubenberger, S., Yaron, O., Anderson, J. P., Ashall, C., Balland, C., Baltay, C., Barbarino, C., Bauer, F. E., Baumont, S., Bersier, D., Blagorodnova, N., Bongard, S., Botticella, M. T., Bufano, F., Bulla, M., Cappellaro, E., Campbell, H., Cellier-Holzem, F., Chen, T.-W., Childress, M. J., Clocchiatti, A., Contreras, C., Dall'Ora, M., Danziger, J., de Jaeger, T., De Cia, A., Della Valle, M., Dennefeld, M., Elias-Rosa, N., Elman, N., Feindt, U., Fleury, M., Gall, E., Gonzalez-Gaitan, S., Galbany, L., Morales Garoffolo, A., Greggio, L., Guillou, L. L., Hachinger, S., Hadjiyska, E., Hage, P. E., Hillebrandt, W., Hodgkin, S., Hsiao, E. Y., James, P. A., Jerkstrand, A., Kangas, T., Kankare, E., Kotak, R., Kromer, M., Kuncarayakti, H., Leloudas, G., Lundqvist, P., Lyman, J. D., Hook, I. M., Maguire, K., Manulis, I., Margheim, S. J., Mattila, S., Maund, J. R., Mazzali, P. A., McCrum, M., McKinnon, R., Moreno-Raya, M. E., Nicholl, M., Nugent, P., Pain, R., Pignata, G., Phillips, M. M., Polshaw, J., Pumo, M. L., Rabinowitz, D., Reilly, E., Romero-Cañizales, C., Scalzo, R., Schmidt, B., Schulze, S., Sim, S., Sollerman, J., Taddia, F., Tartaglia, L., Terreran, G., Tomasella, L., Turatto, M., Walker, E., Walton, N. A., Wyrzykowski, L., Yuan, F., and Zampieri, L., "PESSTO: survey description and products from the first data release by the Public ESO Spectroscopic Survey of Transient Objects," $A \& A$ 579, A40 (July 2015).

[14] Atwood, W. B., Abdo, A. A., Ackermann, M., Althouse, W., Anderson, B., Axelsson, M., Baldini, L., Ballet, J., Band, D. L., Barbiellini, G., and et al., "The Large Area Telescope on the Fermi Gamma-Ray Space Telescope Mission," ApJ 697, 1071-1102 (June 2009).

[15] Guidorzi, C., Monfardini, A., Gomboc, A., Mottram, C. J., Mundell, C. G., Steele, I. A., Carter, D., Bode, M. F., Smith, R. J., Fraser, S. N., Burgdorf, M. J., and Newsam, A. M., "The Automatic Real-Time Gamma-Ray Burst Pipeline of the 2 m Liverpool Telescope," PASP 118, 288-296 (Feb. 2006).

[16] Abbott, B. P., Abbott, R., Abbott, T. D., Abernathy, M. R., Acernese, F., Ackley, K., Adams, C., Adams, T., Addesso, P., Adhikari, R. X., and et al., "Localization and broadband follow-up of the gravitational-wave transient GW150914," ArXiv e-prints (Feb. 2016).

[17] Copperwheat, C. M., Steele, I. A., Bates, S. D., Smith, R. J., Bode, M. F., Baker, I., Peacocke, T., and Thomson, K., "Liverpool Telescope 2: a new robotic facility for time domain astronomy in 2020+," in [Society of Photo-Optical Instrumentation Engineers (SPIE) Conference Series], Society of Photo-Optical Instrumentation Engineers (SPIE) Conference Series 9145, 11 (July 2014).

[18] Vernet, J., Dekker, H., D'Odorico, S., Kaper, L., Kjaergaard, P., Hammer, F., Randich, S., Zerbi, F., Groot, P. J., Hjorth, J., Guinouard, I., Navarro, R., Adolfse, T., Albers, P. W., Amans, J.-P., Andersen, J. J., Andersen, M. I., Binetruy, P., Bristow, P., Castillo, R., Chemla, F., Christensen, L., Conconi, P., Conzelmann, R., Dam, J., de Caprio, V., de Ugarte Postigo, A., Delabre, B., di Marcantonio, P., Downing, M., Elswijk, E., Finger, G., Fischer, G., Flores, H., François, P., Goldoni, P., Guglielmi, L., Haigron, R., Hanenburg, H., Hendriks, I., Horrobin, M., Horville, D., Jessen, N. C., Kerber, F., Kern, L., Kiekebusch, M., Kleszcz, P., Klougart, J., Kragt, J., Larsen, H. H., Lizon, J.-L., Lucuix, C., Mainieri, V., Manuputy, R., Martayan, C., Mason, E., Mazzoleni, R., Michaelsen, N., Modigliani, A., Moehler, S., Møller, P., Norup Sørensen, A., Nørregaard, P., Péroux, C., Patat, F., Pena, E., Pragt, J., Reinero, C., Rigal, F., Riva, M., Roelfsema, R., Royer, F., Sacco, G., Santin, P., Schoenmaker, T., Spano, P., Sweers, E., Ter Horst, R., Tintori, M., Tromp, N., van Dael, P., van der Vliet, H., Venema, L., Vidali, M., Vinther, J., Vola, P., Winters, R., Wistisen, D., Wulterkens, G., and Zacchei, A., "X-shooter, the new wide band intermediate resolution spectrograph at the ESO Very Large Telescope," A $\& A$ 536, A105 (Dec. 2011). 
[19] Piascik, A. S., Steele, I. A., Bates, S. D., Mottram, C. J., Smith, R. J., Barnsley, R. M., and Bolton, B., "SPRAT: Spectrograph for the Rapid Acquisition of Transients," in [Society of Photo-Optical Instrumentation Engineers (SPIE) Conference Series], Society of Photo-Optical Instrumentation Engineers (SPIE) Conference Series 9147, 8 (July 2014).

[20] Greiner, J., Bornemann, W., Clemens, C., Deuter, M., Hasinger, G., Honsberg, M., Huber, H., Huber, S., Krauss, M., Krühler, T., Küpcü Yoldaş, A., Mayer-Hasselwander, H., Mican, B., Primak, N., Schrey, F., Steiner, I., Szokoly, G., Thöne, C. C., Yoldaş, A., Klose, S., Laux, U., and Winkler, J., "GROND: a 7-Channel Imager," PASP 120, 405-424 (Apr. 2008).

[21] O'Brien, K., Thatte, N., and Mazin, B., "KIDSpec: an MKID based medium resolution integral field spectrograph," in [Ground-based and Airborne Instrumentation for Astronomy V], SPIE 9147, 91470G (July 2014). 